\title{
Helioseismology
}

\author{
Ronald L. Gilliland \\ Space Telescope Science Institute 1 , 3700 San Martin Drive, Baltimore, \\ $M D 21218 U S A$
}

\begin{abstract}
Observations of solar $p$-mode oscillations over the last two decades have provided a fundamentally new and unique means of probing the Sun's interior structure. The history of understanding solar oscillations as trapped normal-mode pulsations will be briefly discussed. The large number of excited modes seen in the Sun have been used to constrain the solar core structure, determine the convection zone depth and to fix the variation of angular velocity with depth and latitude. Standard stellar structure and evolution theory does remarkably well in matching the helioseismic results. Further work promises to reveal the solar helium abundance. Upcoming network and space observations should provide the stability and resolution necessary to return detailed information on near-surface structures associated with solar activity.
\end{abstract}

\section{Introduction and history}

Helioseismology is the study of the solar interior conducted by inferences from properties of global oscillation modes seen at the solar surface. In the early 1960s the first evidence for ubiquitous surface oscillations was reported by Leighton, Noyes and Simon (1962) from two-dimensional Doppler measurements. The now universally adopted explanation of the solar motions as trapped, normal-mode, acoustic-cavity oscillations was first put forward by Ulrich (1970) and Leibacher and Stein (1971). In the 1970s observational data improved dramatically and the theoretical basis for global oscillation modes was solidified, but helioseismology as such did not yet exist. In particular the observational work of Deubner (1975), in conjunction with application of new analysis techniques established the photospheric oscillations as the fundamental modes of standing acoustic waves trapped below the photosphere. A recurring theme of helioseismology has been how well standard theory predicts general oscillation characteristics; this was already seen by Deubner (1975): "we find that the solutions of Ulrich (1970) agree with the observed ridges in all detail to an embarrassing extent." The 1980s saw rapid growth in the observational capabilities (full disk Doppler observations by Claverie, et al. 1979, and Grec, et al. 1980 are of special note), applications of theory to exploit the new data to learn more about the solar

\footnotetext{
'Operated by the Association of Universities for Research in Astronomy, Inc., under contract with the National Aeronautics and Space Administration.
} 
interior and a rapidly burgeoning number of investigators and publications. Today, helioseismology is in its most productive years with fundamental advances, planned now for a decade, in observational capability just months away (GONG and $\mathrm{SOHO}$ ).

I will provide brief and very incomplete introductions to the theory and observations of solar oscillations in $\S 2$. Most of my presentation will examine early successes of helioseismology in constraining global properties of the Sun (§3), and a discussion in $\S 4$ of some matters of detail that can be explored. A final section will offer thoughts on what is still to come.

\section{Background on theory and observations}

The physics of pressure wave propagation in an atmosphere has been understood since early in the century (Lamb 1908). In particular an acoustic wave can propagate only at frequencies higher than $c /(2 H)$, the acoustic cut-off frequency which varies as the inverse square root of temperature, where $c$ is the sound speed and $H$ is the density scale height. Consider the situation of approaching the outer layers of the Sun from within for a spectrum of acoustic wave frequencies. In the solar atmosphere near the top of the convection zone the temperature drops rapidly near an acoustic cut-off frequency corresponding to about 200 seconds. Waves with periods longer than this cannot propagate beyond this region and are effectively reflected back into the interior. Waves at higher frequencies can escape freely from the photosphere.

Wavelengths for the acoustic waves may be determined from a dispersion relation for the modes. Near the upper reflection point the radial wavelength is large compared to the horizontal wavelength and propagation is nearly vertical. An acoustic wave propagating toward the interior encounters higher sound speeds, a decreasing vertical to horizontal wavelength and is effectively refracted back toward the surface. This lower turning point thus fixes the size of the acoustic cavity for a given mode. Properties of the modes become clear once an appropriate mathematical description, decomposition into spherical harmonics, is adopted and the wave equation for adiabatic oscillations is worked through. Excellent discussions have been provided by Deubner \& Gough (1984) and Christensen-Dalsgaard (1988). The normal modes of oscillation are characterised by three wave numbers: the order, $n$, giving the number of nodes in the radial direction, $l$ is the degree which specifies horizontal scales, and with breaking of spherical symmetry (e.g., as with rotation) $m$ corresponds to the number of nodes around the equator. The spatial and temporal wave perturbations are expressed:

$$
\xi_{n l m}(r, \theta, \phi, t)=\xi_{n l}(r) Y_{l}^{m}(\theta, \phi) e^{-i \omega_{n l m} t},
$$

where $\xi$ is the scalar perturbation (e.g., radial displacement); $r, \theta, \phi$, and $t$ are the radial coordinate, the colatitude, longitude, and time, respectively, and the $Y_{l}^{m}$ are spherical harmonic functions. The amplitudes of solar oscillations are very small and linear perturbation analyses (Unno et al. 1989) on the usual equations of stellar structure suffice to derive theoretical estimates of mode properties. 


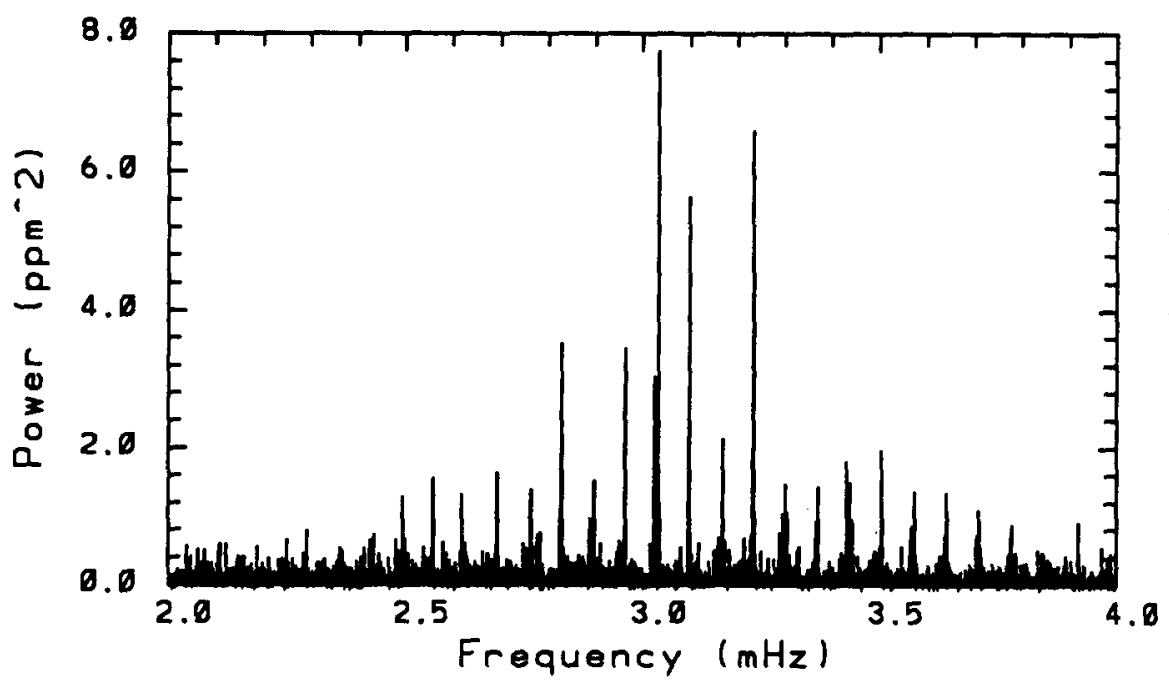

Figure 1. Power spectrum of one month of disk-integrated solar intensity measured with the green channel of the IPHIR experiment (Toutain \& Fröhlich 1992).

Solar oscillations have been studied by two primary approaches: 1) Measurements of surface velocity through spectroscopic monitoring of Doppler shifts in atmospheric absorption features. 2) Measurement of intensity variations through photometric monitoring. The solar oscillations consist of literally millions of independent modes, with velocity amplitudes peaking at about $15 \mathrm{~cm} / \mathrm{s}$.

A few of the lowest degree oscillations $(l \leq 3)$ may be observed with light integrated over the full solar disk. Such observations can be obtained with relatively simple equipment, since no spatial resolution is required. However, with amplitudes of only $15 \mathrm{~cm} / \mathrm{s}$, or $\delta L / L \sim 3 \mathrm{ppm}$ (parts per million) the requisite precision is extreme. Figure 1 shows the power spectrum of solar $p$ modes from full-disk data; with spatial resolution the number of observable modes rises dramatically (see, e.g., Libbrecht \& Woodard 1990).

As with the theory of solar oscillations, there exist many excellent reviews of observing techniques, and it is unlikely that I could add in either a qualitatively, or even a quantitatively meaningful way to these. The review by Brown (1988) thoroughly discusses the observational challenges, the several methods in use, and includes in an integral way a consideration of mathematical analysis techniques that must be used to extract useful information from the observations. 


\section{Constraining the global solar interior}

Only within the past decade have the observations of solar oscillations reached a sufficient level of accuracy to support seismology inferences. In this section I review three areas where helioseismology has provided useful constraints and fundamentally new results. The review by Gough \& Toomre (1991) provides a thorough introduction to helioseismic applications.

\subsection{Core structure}

The solar neutrino problem, the observation of a factor of 2-3 fewer than theoretically expected neutrinos from the fusion reactions that power the Sun, has been a significant problem for two decades (see, e.g., Bahcall 1989). Until quite recently most of the solar neutrinos that could be detected from the ground came from a very (exponent of order 15) temperature sensitive, but energetically unimportant, tertiary reaction. Over the past two decades dozens of theories have been put forth to provide modifications to solar models based on best estimates of applicable standard physics, in order to explain the neutrino deficit.

A core temperature decrease of just a few percent below the best estimate solar value would suffice to explain the low neutrino flux. Any modification of the central temperature (e.g., through opacity modifications, or assumed composition differences) results in a change of sound speed. As one might imagine, acoustic wave frequencies are sensitive to integrals of the sound speed over the acoustic cavity. Although mode frequencies primarily reflect conditions throughout the Sun, differences of frequencies for the low $l$-degree modes that penetrate most deeply do provide sensitivity to core conditions. Despite some early indications favouring one class of non-standard solar models (with weakly interacting massive particles to effectively provide a lowered core opacity), helioseismology now substantially supports the standard solar model (Dziembowski et al. 1994), suggesting that an explanation of the neutrino problem is more likely to follow from nuclear physics, than from non-standard physics.

\subsection{Convection zone depth}

Most aspects of solar and stellar evolution modelling are considered to be robust. An acknowledged weak link, however, in computing stellar models is the almost universally used mixing length theory of convection (with a free parameter - the mixing length to pressure scale height ratio). Detailed inference from solar or stellar evolution theory that relies on assumptions about convection zone structure is therefore particularly suspect. The abundances of lithium and beryllium are of cosmic significance in constraining models of the early universe. Lithium and beryllium are easily destroyed at temperatures of a few million degrees. If the bottom of a stellar convection zone extends beyond these critical temperatures these fragile elements are destroyed. A direct calibration of the solar convection zone depth is thus of broad interest.

The bulk of the solar convection zone is nearly adiabatically stratified. Convection occurs when the actual temperature gradient exceeds the adiabatic value. Convective transport at depth is very efficient and regulates the stratification to be just slightly superadiabatic. Although different solar models can have significantly different convection zone depths depending upon assumptions of condi- 
tions within the convection zone, they are ubiquitous in showing a rapid transition to strong subadiabatic conditions below the convective boundary. Gough (1986) argued that direct inversions for the sound speed would show the convection zone boundary as a clear break in the gradient of the sound speed. The hard part is of course inverting for the sound speed. For our purposes it is sufficient to point out that the mode frequencies may be expressed as an integral function of the sound speed over the radius. Given many separate oscillation mode frequencies it is in principle possible to invert an integral equation to determine $c(r)$. Christensen-Dalsgaard, Gough \& Thompson (1991) have rigourously applied this approach to fix the solar convection zone depth at $0.287 \pm 0.003$ solar radii.

\subsection{Angular velocity with depth and latitude}

The Sun is observed to rotate differentially at the surface as a function of latitude (faster at the equator). The depth dependence of solar rotation is of considerable importance to testing dynamical theories of magnetic field generation. Direct, multi-dimensional numerical models of convection zone dynamics (Glatzmaier 1985; Gilman \& Miller 1986) predicted that rotation within the convection zone would have constant angular velocity on cylinders aligned with the rotation.

As with inversions for the sound speed, I will argue that integral equations involving the rotation profile over radius and latitude can be formulated for the amount of splitting in the $m$-dependence of solar oscillation frequencies (this eq. shows the $r$ dependence only):

$$
\nu_{n l m}=\nu_{n l 0}+\frac{m \beta_{n l}}{2 \pi} \int_{0}^{R *} \Omega(r) K_{n l}(r) d r
$$

where $\beta_{n l}$ is an order of unity correction factor, $\Omega(r)$ is the solar angular velocity, and $K_{n l}$ is a kernel that may be derived from solar models and is proportional to the local energy density of the mode (Libbrecht \& Morrow 1991). A consistent result from such studies (Christensen-Dalsgaard \& Schou 1988, Brown et al. 1989; Dziembowski, Goode, \& Libbrecht 1989; Thompson 1990; Schou \& Brown 1994) has been that the Sun does not show constant rotation on cylinders, but rather the latitude dependence remains essentially that directly visible at the surface throughout the convection zone $\left(r \geq 0.7 R_{\odot}\right)$. (Actually the results are not considered significant above about $0.85 R_{\odot}$.) The radiative interior is found to rotate like a solid body (down to $r \sim 0.5 R_{\odot}$ where the results are considered reliable) with an angular velocity as seen at mid-latitudes on the solar surface.

The inversions are sensitive to the selection of mode frequencies used as well as the errors. Say that one is interested in the solar rotation rate at a specific radius. Many oscillation modes will have turning points above the radius of interest and therefore have no dependence on the quantity of interest. For modes of lower degree with finite eigenfrequencies in the region of interest, there is some response to conditions at the specified radius, but there is also sensitivity to conditions over a broad range of radii. Such inversions suffer from the same ill-conditioning that plagues any deconvolution process, and small amounts of noise can be amplified to mimic physically interesting, but spurious results.

The rotation rate of the solar core has long been a primary goal of helioseismology. Here it is possible to describe the inherent difficulties with some clarity. 
Rotation splits $l$-modes into $m=-l, \ldots, l$ components, the $l=0$ (radial) modes do not respond to rotation directly. The size of the splitting is comparable to the angular rotation rate of the Sun, at a period of 27 days, this corresponds to $\sim 440 \mathrm{nHz}$ if the Sun were to rotate as a solid body. The $l=1$ modes provide the greatest penetration depth and hence sensitivity to core rotation. To resolve a splitting of $440 \mathrm{nHz}$ requires a time series spanning at least one rotation period; to measure the small changes of $\sim 10 \mathrm{nHz}$ that would provide reasonable sensitivity to core rotation requires three years worth of data. At such long baselines two fundamental problems appear: 1) The $p$-modes have finite lifetimes. These generally increase as the frequency (order $n$ ) decreases. Selection of modes in the low frequency tail of the distribution may provide a long lifetime, but these modes have low amplitudes and hence provide lower S/N. 2) As will be discussed further in $\$ 5.1$ model frequencies are known to change over the 11 year solar cycle. The amplitude of change for $l=1$ is comparable to the change of rotational splitting from a rapidly rotating core. Determinations of core rotation $\left(r<0.2 R_{\odot}, m<0.3 M_{\odot}\right)$ have shown large variations, but generally indicate a core that rotates at 2 to 4 times the surface rate (Jiménez, et al. 1994).

\section{Down to details}

Developments over the past decade in helioseismology have shown an increasing range of interesting results. In this section I discuss the surprising extent to which solar oscillation frequencies vary over the sunspot cycle. The physics responsible for solar oscillations may be elucidated through study of waves with frequencies higher than the photospheric acoustic cut-off frequency; such waves can propagate out of the source region and may provide direct information on sources. The local properties of sound waves are perturbed by inhomogeneities in the atmosphere; it may be possible to determine the structure of sunspots at depth through monitoring of such local oscillation changes.

\subsection{Zeroing in on acoustic sources}

I have earlier argued that acoustic waves with frequencies less than $\sim 5.3 \mathrm{mHz}$ (3 minutes) suffer near total reflection in layers near the photosphere; these trapped modes exhibit the discrete frequencies as determined by the eigenmodes of the resonant cavity. The trapped modes can provide information on structural properties of the acoustic cavity, but their source is hidden. Sound waves generated in the interior at frequencies above $5.3 \mathrm{mHz}$ are free to propagate through the photosphere and can therefore exist at a continuum of frequencies. Observations of these oscillations might be expected to provide rather direct inferences to be drawn about excitation sources.

Observations extending well above $5.3 \mathrm{mHz}$ do not show a continuous distribution of frequencies, but rather a decaying sinusoidal envelope of power $(\sim$ $150 \mu \mathrm{Hz}$ between peaks). Kumar \& Lu (1991) and Kumar (1994) have provided an elegant explanation. If the acoustic sources are restricted to a fairly narrow depth, an interference pattern is expected from waves propagating directly toward the observer and those which started toward the interior, but were refracted back toward the surface. The quantitative result is that the sources are $140 \pm 60 \mathrm{~km}$ (about 1.5 density scale heights) below the photosphere. In a 
general sense this is consistent with the expectation that $p$-modes result from stochastic excitation by near-surface turbulent convection (see Goldreich et al. 1994 for the latest theory here).

\subsection{Solar cycle changes}

Woodard \& Noyes (1985) noted an apparent decrease of oscillation frequencies in the $l=0-2 p$-modes detected by the Solar Maximum Mission spacecraft as the Sun transitioned from high to low solar activity. Since the frequencies of solar acoustic modes depend upon the interior structure of the Sun, it would be expected that changes of interior conditions associated with the solar cycle would induce changes in the $p$-modes. Over the past decade a consistent picture of frequency changes involving a large range of $l$-modes has begun to emerge; the inferences to be drawn concerning changes to interior properties have not yet become clear.

The best observational evidence for the $l=0-2$ modes that can probe the solar core follow from resonant scattering spectrometer studies conducted by the Birmingham group (Elsworth et al. 1994). Data for the oscillation frequencies span 1981 to 1994 and thus cover more than one solar cycle; when the mean frequency of the 24 strongest modes are tracked a remarkably strong correlation with solar activity emerges such that frequencies are lower by $\sim 0.5 \mu \mathrm{Hz}$ (out of $3,000 \mu \mathrm{Hz}$ ) at solar minimum relative to the maximum.

Observation for higher harmonics, $20 \leq l \leq 60$ spanning 1984 to 1990 (minimum to maximum) from Fourier Tachometer observations are reported by Bachmann \& Brown (1994). The frequency changes found for these higher degree modes are very similar to those for the low-degree modes. Nearly equal response across a wide range of $l$ suggests that the causal perturbations are in the surface layers of the Sun. A more detailed constraint on this has been provided by Goldreich et al. (1991) who consider frequency shifts as a function of magnetic field stresses in the outer layers of the Sun. Inherent in this analysis is a comparison to the distribution of frequency change with mode order, $n$, which is much smaller at low frequency (Libbrecht \& Woodard 1990). Goldreich et al. (1991) find that a variation of the mean magnetic stress at a level of $1 \%$ of the gas pressure is sufficient to explain the variations. This theory seems largely confirmed by Woodard et al. (1991) who find tight correlation of solar $p$-mode frequency changes with measured surface magnetic field strength on time scales as short as one month. However, a loose end remains that current theory would predict an order $1 \%$ change of solar luminosity (observations show changes 10 times smaller) associated with this.

\subsection{Probing sunspots}

Attempts to determine the structure of individual sunspots at depth through helioseismology is indicative of the increasingly more detailed applications in this field. Just as a local blockage on the surface of a pond can create interesting patterns as surface ripples propagate past it, one would expect the local properties of $p$-modes to be altered as they propagate through inhomogeneities in the solar atmosphere. By examining the incoming and outgoing waves associated with an individual sunspot it is possible to set constraints on the sunspot structure. 
Observations of $p$-modes on the restricted spatial scales and temporal lifetimes of individual sunspots is quite challenging, nonetheless independent studies (Braun et al. 1992; Bogdan et al. 1993) are now obtaining reasonably consistent results in terms of the observed perturbations to $p$-mode characteristics as a function of frequency and wavelength. The theory of how sunspots are expected to influence the acoustic waves is even more challenging (e.g., Bogdan 1992) and it is fair to say that this promising field has yet to experience a closure between theory and observations.

\section{Still to come}

Helioseismology is a rapidly expanding field of investigation. Both theory and observations have made complementary and fundamental advances in recent years. The near future promises an even more quickening pace thanks to the completion of long planned for experiments.

\subsection{New experiments: GONG and SOHO}

Both the Global Oscillation Network Group (GONG) Project and the Solar and Heliospheric Observatory (SOHO) have been in active planning for over a decade. GONG should have its six longitudinally distributed observing sites deployed in 1995 and SOHO is scheduled for launch in 1995. The two experiments are complementary and each promises fundamental, qualitative advances over previous observations.

GONG (see, e.g., Harvey et al. 1993) will provide nearly continuous coverage of the Sun for 3-11 years through the use of longitude-redundant siting of identical instruments. Each instrument employs a Fourier Tachometer to provide spatially resolved Doppler images at a cadence of one minute. The data resulting from GONG will surpass previous experiments by orders of magnitude in providing the ability to squeeze information out of the multitude of low amplitude solar $p$-modes.

SOHO (see, e.g., Domingo 1988) will be launched by ESA into a continuous Sun-viewing orbit, currently scheduled for October 1995. SOHO will carry several instruments, three of which are for helioseismology. A high resolution spectrometer (GOLF) and solar irradiance monitor (VIRGO) will provide complementary velocity and intensity measurements at low spatial resolution for the study of long period, low degree modes. The solar oscillation imager (SOI) will exploit seeing-free imaging of the full solar disk at high spatial resolution to study oscillation modes at very high degree.

It is expected that both GONG and SOHO will provide impressive new results, particularly for probing those parts of the Sun requiring long-stable measurements (the core), or very high spatial resolution (near surface conditions).

\subsection{New theoretical frontiers}

Making full use of the high quality data about to come on line from GONG and SOHO will require continuing advances, particularly in the area of inversions. Most helioseismology to date has exploited the global, normal-mode character- 
istic of solar oscillations. Increasingly studies of local characteristics are coming into consideration (e.g., as in probing sunspots). A fundamentally new approach of "time-distance helioseismology" (Duvall et al. 1993; D'Silva \& Duvall 1995) may provide a means of probing the near-surface regions through measuring the travel times and distances of solar acoustic waves.

The solar helium abundance is of particular interest because of cosmological and galactic evolution implications. Fixing the helium abundance relies on helioseismic inversions for which the sensitivity of model details of the equation of state used are particularly severe. Thus, while solutions exist for the solar helium abundance (see Antia \& Basu 1994 and references therein) I have chosen to list this as a work in progress, where new developments in theory are likely to still be important.

\subsection{Asteroseismology}

Solar-like oscillations have been unambiguously detected on only one star (the Sun) despite many attempts by several groups over the past decade (see Brown \& Gilliland 1994 for a thorough review). Although, perhaps the next talk (Bedding 1995 ) in this conference will provide such a detection. The study of low-degree $p$-modes on other stars, although challenging observationally, will surely become a fundamentally important new area of stellar astrophysics within a decade.

\section{References}

Antia, H.M., \& Basu, S. 1994, ApJ, 426, 801

Bachmann, K.T., \& Brown, T.M. 1993, ApJ, 411, L45

Bahcall, J.N. 1989, Neutrino Astrophysics, (Cambridge University Press: New York).

Bedding, T.R. 1995, this volume

Bogdan, T.J. 1992, in Sunspots: Theory and Observations, ed. J.H. Thomas \& N.O. Weiss (Dordrecht: Kluwer), 345

Bogdan, T.J., Brown, T.M., Lites, B.W., \& Thomas, J.H. 1993, ApJ, 406, 723

Braun, D.C., Duvall, T.L., Jr., LaBonte, B.J., Jefferies, S.M., Harvey, J.W., \& Pomerantz, M.A. 1992, ApJ, 391, L113

Brown, T.M. 1988, in Advances in Helio- and Asteroseismology, ed. J. ChristensenDalsgaard \& S. Frandsen, (D. Reidel: Dordrecht), 453

Brown, T.M., Christensen-Dalsgaard, J., Dziembowski, W.A., Goode, P., Gough, D.O., \& Morrow, C.A. 1989, ApJ, 343, 526

Brown, T.M., \& Gilliland, R.L. 1994, ARA\&A, 32, 37

Christensen-Dalsgaard, J. 1988, in Advances in Helio- and Asteroseismology, ed. J. Christensen-Dalsgaard \& S. Frandsen, (D. Reidel: Dordrecht), 3

Christensen-Dalsgaard, J., \& Schou, J. 1988, in ESA SP-286, Seismology of the Sun and Sun-like Stars, ed. V. Domingo \& E.J. Rolfe (Nordwijk: ESTEC), 149

Christensen-Dalsgaard, J., Gough, D.O., \& Thompson, M.J. 1991, ApJ, 378, 413 
Claverie, A., Isaak, G.R., McLeod, C.P., van der Raay, H.B., \& Roca Cortez, T. 1979, Nature, 282, 591

Deubner, F.-L. 1975, AA, 44, 371

Deubner, F.-L., \& Gough, D.O. 1984, ARA\&A, 22, 593

Domingo, V. 1988, in Advances in Helio- and Asteroseismology, ed. J. ChristensenDalsgaard \& S. Frandsen, (D. Reidel: Dordrecht), 545

D'Silva, S., \& Duvall, T.L., Jr. 1995, ApJ, 438, 454

Duvall, T.L., Jr., Jefferies, S.M., Harvey, J.W., \& Pomerantz, M.A. 1993, Nature, 362,430

Dziembowski, W.A., Goode, P.R., \& Libbrecht, K.G. 1989, ApJ, 337, L53

Dziembowski, W.A., Goode, P.R., Pamyatnykh, A.A., \& Sienkiewicz, R. 1994, ApJ, 432, 417

Elsworth, Y., Howe, R., Isaak, G.R., McLeod, C.P., Miller, B.A., New, R., Speake, C.C., \& Wheeler, S.J. 1994, ApJ, 434, 801

Gilman, P.A., \& Miller, J. 1986, ApJS, 61, 585

Glatzmaier, G. 1985, ApJ, 291, 300

Goldreich, P., Murray, N., Willette, G., \& Kumar, P. 1991, ApJ, 370, 752

Goldreich, P., Murray, N., \& Kumar, P. 1994, ApJ, 424, 466

Gough, D.O. 1986, in Seismology of the Sun and the Distant Stars, ed. D.O. Gough (Dordrecht: Reidel), 125

Gough, D., \& Toomre, J. 1991, ARA\&A, 29, 627

Grec, G., Fossat, E., \& Pomerantz, M. 1980, Nature, 288, 541

Harvey, J., Hill, F., Kennedy, J., \& Leibacher, J. 1993, in GONG 1992: Seismic Investigation of the Sun and Stars, ed. T.M. Brown, ASP Conf. Ser., 42, 397

Jiménez, A., Herández, F.P., Claret, A., Pallé, P.L., Régulo, C., \& Roca Cortés, T. 1994, ApJ, 435, 874

Kumar, P., \& Lu, E. 1991, ApJ, 375, L35

Kumar, P. 1994, ApJ, 428, 827

Lamb, H. 1908, Proc. London, Math. Soc.(2), VII, 122

Leibacher, J., \& Stein, R.F. 1971, Astrophys. L., 7, 191

Leighton, R.B., Noyes, R.W., \& Simon, G.W. 1962, ApJ, 135, 474

Libbrecht, K.G., Woodard, M. 1990, Nature, 345, 779

Libbrecht, K.G., \& Morrow, C.A. 1991, in Solar Interior and Atmosphere, ed. A.N. Cox, W.C. Livingston, \& M.S. Matthews, (Tucson: Univ. Ariz. Press), 479.

Schou, J., \& Brown, T.M. 1994, ApJ, 434, 378

Thompson, M.J. 1990, Solar Phys., 125, 1

Toutain, T., \& Fröhlich, C. 1992, AA, 257, 287

Ulrich, R.K. 1970, ApJ, 162, 993

Unno, W., Osaki, Y., Ando, H., Saio, H., \& Shibahashi, H. 1989. Nonradial Oscillations of Stars, (Tokyo: Univ. Tokyo Press), 2nd ed.

Woodard, M.F., \& Noyes, R.W. 1985, Nature, 318, 449 
Woodard, M.F., Kuhn, J.R., Murray, N., \& Libbrecht, K.G. 1991, ApJ, 373, L81

\section{Discussion}

Christensen-Dalsgaard: a) A comment on the core rotation. Measurement of low- $\ell$ splitting is very difficult, as the peaks often partially overlap. However, recent data from the BISON group shows clearly separated peaks for $\ell=1$ and 2. This splitting is consistent with constant rotation in the interior.

b) It is really a big surprise that the standard models are so close to the observations. However, to many of us it was a surprise that He settling is so important in getting the models to agree with the observations. This was regarded as a very non-standard effect until a few years ago, despite pioneering work at Los Alamos.

c) On observational and theoretical accuracy: In much of the Sun, the difference between observation and theory exceeds the observational errors by more than an order of magnitude. Hence, theory has a long way to go. On the other hand, better data are definitely needed, particularly with regards to the core and near-surface structure.

Goode: Recent observational results indicate that solar oscillations are not excited by convective drumming from below, but rather by the catastrophic cooling and subsequent collapse of the surface.

Christensen-Dalsgaard: It is very interesting that observations now indicate that excitation is dominated by the strong downdrafts. However, as a clarification it should be mentioned that this is really a refinement of the basic convective excitation picture, rather than a drastically new approach. 